\title{
Candidate Screening of the TRPC3 Gene in Cerebellar Ataxia
}

\author{
Esther B. E. Becker • Brent L. Fogel • Sanjeev Rajakulendran • Anna Dulneva • \\ Michael G. Hanna • Susan L. Perlman • Daniel H. Geschwind • Kay E. Davies
}

Published online: 16 February 2011

(C) The Author(s) 2011. This article is published with open access at Springerlink.com

\begin{abstract}
The hereditary cerebellar ataxias are a diverse group of neurodegenerative disorders primarily characterised by loss of balance and coordination due to dysfunction of the cerebellum and its associated pathways. Although many genetic mutations causing inherited cerebellar ataxia have been identified, a significant percentage of patients remain whose cause is unknown. The transient receptor potential (TRP) family member TRPC3 is a non-selective cation channel linked to key signalling pathways that are affected in cerebellar ataxia. Furthermore, genetic mouse models of TRPC3 dysfunction display cerebellar ataxia, making the TRPC3 gene an excellent candidate for screening ataxic patients with unknown genetic aetiology. Here, we report a genetic screen for TRPC 3 mutations in a cohort of 98 patients with genetically undefined late-onset cerebellar ataxia and further ten patients with undefined episodic ataxia. We identified a number of variants but no causative mutations in TRPC3. Our findings suggest that mutations in TRPC3 do not significantly contribute to the cause of late-onset and episodic human cerebellar ataxias.
\end{abstract}

E. B. E. Becker $(\bowtie) \cdot$ A. Dulneva $\cdot$ K. E. Davies

MRC Functional Genomics Unit, Department of Physiology,

Anatomy and Genetics, University of Oxford,

South Parks Road,

Oxford OX1 3QX, UK

e-mail: esther.becker@dpag.ox.ac.uk

B. L. Fogel $\cdot$ S. L. Perlman • D. H. Geschwind

Department of Neurology, Program in Neurogenetics,

David Geffen School of Medicine,

University of California at Los Angeles,

2309 Gonda Bldg., 695 Charles E. Young Dr. South,

Los Angeles, CA 90095-1761, USA

S. Rajakulendran $\cdot$ M. G. Hanna

MRC Centre for Neuromuscular Diseases, Institute of Neurology,

University College London,

Queen Square,

London WC1N 3BG, UK
Keywords Hereditary ataxia - Cerebellar dysfunction . Neurodegeneration - Transient receptor potential channel

\section{Introduction}

The hereditary ataxias are a clinically and genetically heterogeneous group of inherited neurodegenerative disorders with ataxia as the predominant symptom. The hereditary ataxias include the autosomal recessive ataxias, the dominantly inherited spinocerebellar ataxias (SCA) and episodic ataxias (EA) and the X-linked ataxias [12]. Although the causative mutations have been identified in a number of ataxia subtypes, the underlying genetic defects for many forms of hereditary ataxia remain unknown.

The transient receptor potential (TRP) channel family member TRPC3 is a non-selective cation channel, which is highly enriched in the Purkinje cells of the cerebellum [2, 8]. TRPC 3 is a key mediator of metabotropic glutamate receptor (mGluR)-dependent synaptic transmission in the Purkinje cells [8] and has also been implicated in cerebellar development $[2,10]$. Interestingly, TRPC 3 is associated with several proteins that are linked to human cerebellar ataxia, including mGluR1, the IP3 receptor and protein kinase $\mathrm{C} \gamma(\mathrm{PKC} \gamma)[8,14]$. In vitro studies have shown that TRPC 3 is a substrate of $\mathrm{PKC} \gamma$, and that mutants of PKC $\gamma$ causing SCA14 fail to phosphorylate TRPC3, resulting in dysfunctional calcium homeostasis [1]. Furthermore, in a mouse model of SCA1, TRPC3 is downregulated before the onset of degeneration [11]. Notably, genetic mouse models of TRPC 3 dysfunction exhibit cerebellar ataxia [2, 8]. Collectively, these studies point to an important role of TRPC 3 in the pathways leading to cerebellar ataxia in mice and humans and suggest TRPC 3 as a promising candidate gene for hereditary forms of human cerebellar ataxia. 
Table 1 Characteristics of the patient populations studied

\begin{tabular}{lccccccc}
\hline Number & Age at exam (years) & Female (\%) & Cerebellar only (\%) & SCA-like (\%) & Spasticity (\%) & Episodic (\%) & MSA-like (\%) \\
\hline 98 & $55.5 \pm 15.8$ & 57.1 & 28.6 & 37.8 & 12.2 & 8.2 & 13.3 \\
10 & $25 \pm 14$ & 33.3 & 0 & 0 & 0 & 100 & 0 \\
\hline
\end{tabular}

Ninety-eight patients presented with late-onset gait and appendicular ataxia. Additional phenotypic features include involvement of spinocerebellar pathways and/or peripheral neuropathy (SCA-like), increased lower and/or upper extremity tone (spasticity), fluctuating periods of ataxia (episodic) or autonomic neuropathy and/or parkinsonism not diagnostic for multiple system atrophy (MSA-like). A further panel of ten patients presented with episodic ataxia clinically similar to EA2

Here, we report a mutation screen of the human TRPC3 gene in a cohort of 108 patients with suspected but, as yet, undetermined genetic forms of ataxia.

\section{Subjects and Methods}

\section{Subjects}

Ninety-eight samples were from patients with late-onset cerebellar ataxia seen at a large tertiary referral centre. Patients had sporadic onset or did not have a clearly defined family history of ataxia. Evaluation was performed for acquired and hereditary causes of late-onset cerebellar ataxia according to established clinical protocols [7], and patients did not have a defined genetic diagnosis at the time of screening. Prior genetic analysis was performed individually based on phenotype but, in general, minimal analysis included screening for SCA1, SCA2, SCA3, SCA6, SCA7 and FXN repeat expansions. Patient consent was obtained and DNA was extracted from peripheral blood leukocytes using the Gentra Puregene Blood Kit (Qiagen). All human subject research protocols were approved by the UCLA Institutional Review Board and comply with the current laws of the United States.

DNA samples from ten individuals with clinical features consistent with episodic ataxia type 2 (EA2) were obtained with informed consent. These patients had been previously sequenced for mutations in the coding and flanking intronic regions of $C A C N A 1 A$ and no disease-causing mutations were identified. Ethical approval was obtained from the UCLH ethics committee. DNA from 96 healthy adults (European Collection of Cell Cultures) were screened as controls.

\section{TRPC3 Sequence Analysis}

Patient genomic DNA extracted from blood was amplified using standard PCR techniques with primers for all coding regions of TRPC3 (exons 1-11 of the TRPC3-001 transcript according to the Ensembl genome browser 58) and an average of 80 nucleotides of flanking intronic regions. All amplicons were sequenced using Big Dye 3.1 dideoxy terminator methods (Applied Biosystems) using the amplification primers. To confirm sequence variants, the original DNA stocks were reamplified and re-sequenced. Sequence analysis was performed using 4Peaks software (Mekentosj B.V.). Exonic splicing enhancer (ESE) motifs were predicted using ESEfinder 3.0 (Cold Spring Harbor Laboratory) and RESCUE-ESE [6].

\section{Results}

To identify possible mutations in TRPC3, we screened all 11 coding exons and flanking exon/intron boundaries of this gene in 98 patients with undiagnosed late-onset cerebellar ataxia. Previous work in mice had suggested that TRPC3 mutations cause a dominant pure cerebellar phenotype [2],

Table 2 Identified genetic variants in TRPC3

\begin{tabular}{|c|c|c|c|c|c|}
\hline RefSNP & Variation & Exon & AA change & Genotype frequency & Genotype frequency in population \\
\hline \multirow[t]{2}{*}{ rs13121031 } & c. $78 \mathrm{C}>\mathrm{G}$ & 1 & p.Ala26Ala & $0.148(\mathrm{G} / \mathrm{C})$ & $0.183(\mathrm{G} / \mathrm{C})$ \\
\hline & c.585G $>A$ & 1 & p.Lys195Lys & $0.009(\mathrm{C} / \mathrm{T})$ & $\mathbf{n} / \mathbf{a}$ \\
\hline \multirow[t]{3}{*}{ rs11732666 } & c. $2199 \mathrm{G}>\mathrm{A}$ & 8 & p.Arg733Arg & $0.352(\mathrm{C} / \mathrm{T})$ & $0.5(\mathrm{C} / \mathrm{T})$ \\
\hline & & & & $0.111(\mathrm{~T} / \mathrm{T})$ & $0.1(\mathrm{~T} / \mathrm{T})$ \\
\hline & c. $2271 \mathrm{~A}>\mathrm{G}$ & 9 & p.Ser757Ser & $0.009(T / C)$ & $\mathbf{n} / \mathbf{a}$ \\
\hline rs61741700 & c. $2451 \mathrm{~A}>\mathrm{G}$ & 10 & p.Glu817Glu & $0.019(\mathrm{C} / \mathrm{T})$ & $\mathrm{n} / \mathrm{a}$ \\
\hline
\end{tabular}

Nomenclature is based on the National Center for Biotechnology Information (NCBI) reference sequences NM_003305.2 (mRNA) and NP_003296.1 (protein). SNP reference numbers, genetic variants and population frequency (HapMap-CEU) are liste $\bar{d}$ according to the NCBI dbSNP database. Newly identified variants are indicated in bold

$n / a$ not applicable 
therefore patients with similar phenotypes were selected from a population of undiagnosed ataxia cases at a large tertiary referral centre. The characteristics of this patient population are shown in Table 1. Late-onset cases (onset greater than age 25 years) were chosen as this is the most common presentation of a dominantly inherited cerebellar ataxia [13].

As TRPC3 is an ion channel, it is possible that the human phenotype may demonstrate a paroxysmal course [9]. Accordingly, we investigated whether TRPC3 plays a role in episodic ataxia. We included an additional ten samples from patients with a dominant history of episodic ataxia whose clinical features were most consistent with EA2 in terms of attack precipitants and duration, interictal signs and response to acetazolamide. All ten individuals were initially sequenced for mutations in the CACNA1A gene and found to be negative.

Three known single nucleotide polymorphisms (SNPs) were found in the patient cohort at similar frequencies as published for the HapMap-CEU control population (Table 2). In addition, we identified two novel variants (c.585G $>$ A, c. $2271 \mathrm{~A}>\mathrm{G}$ ), each in a single patient (Table 2). Both of these SNPs result in synonymous changes at the protein level (p.Lys195Lys, p.Ser757Ser). The novel variants were not found in healthy controls. Interestingly, the c. $2271 \mathrm{~A}>\mathrm{G}$ variant in exon 9, which was found in a patient with a complex phenotype of intermittent ataxia and episodic hemiplegia, is predicted to weaken an ESE motif according to bioinformatics calculation. The mutation was also detected in the proband's mother, who had hemiplegic migraine. In an in vitro splicing assay, this putative ESE motif did not have an effect on the splicing of an artificial minigene construct (data not shown). However, this does not rule out the potential importance of this ESE motif for splicing of the full TRPC3 gene in vivo in the patient's nervous system. Unfortunately, this patient was lost to follow-up and material was not available for further splicing analysis.

\section{Discussion}

Genetic mouse models of loss of TRPC3 or dysfunctional TRPC3 result in an ataxic phenotype [2, 8]. Furthermore, several studies have linked TRPC3 to key signalling pathways that are affected in human cerebellar ataxia [1, 14]. TRP family member genes have recently been implicated in human neurological diseases $[4,5]$. We, therefore, hypothesized that mutations in the TRPC 3 gene might underlie previously undiagnosed late-onset forms of human cerebellar ataxia or episodic ataxia. Our findings suggest that mutations in TRPC 3 are not a common cause of late-onset or episodic cerebellar ataxia. We did identify a novel variant in one patient with episodic ataxia that is predicted to alter splicing of the TRPC3 gene. Missplicing of exon 9 would lead to a truncated TRPC 3 protein missing important protein-protein interaction motifs in the intracellular C-terminus, including the CRIB and CaM binding domains [15]. As a consequence, this shorter form of TRPC 3 could act as a dominant negative and impair proper calcium handling in Purkinje cells of the cerebellum.

Despite the absence of TRPC3 mutations in our group of patients, TRPC3 mutations may still contribute to human disease, as it is well-known that genetic mutations do not always produce equivalent phenotypes in mice and humans [3]. Interestingly, TRP gene mutations have recently been associated with human neurological disease [5]. Therefore, future genetic studies in larger patient cohorts and/or different ethnic/geographic populations, as well as in patients with different subtypes of cerebellar ataxia, are necessary to fully elucidate the role TRPC3 may play in human cerebellar ataxia.

Acknowledgements This work was supported by the UK Medical Research Council. E.B. was funded by a fellowship from the International Human Frontier Science Program Organization and an OXION Wellcome Trust training fellowship. Additional support was provided by the National Institute of Mental Health, NIMH K08MH86297 (B.L.F.).

Conflict of Interest Notification Page The authors declare that they have no conflict of interest.

Open Access This article is distributed under the terms of the Creative Commons Attribution Noncommercial License which permits any noncommercial use, distribution, and reproduction in any medium, provided the original author(s) and source are credited.

\section{References}

1. Adachi N, Kobayashi T, Takahashi H, Kawasaki T, Shirai Y, Ueyama T, et al. Enzymological analysis of mutant protein kinase Cgamma causing spinocerebellar ataxia type 14 and dysfunction in Ca2+ homeostasis. J Biol Chem. 2008;283:19854-63.

2. Becker EB, Oliver PL, Glitsch MD, Banks GT, Achilli F, Hardy A, et al. A point mutation in TRPC3 causes abnormal Purkinje cell development and cerebellar ataxia in moonwalker mice. Proc Natl Acad Sci USA. 2009;106:6706-11.

3. Beckers J, Wurst W, de Angelis MH. Towards better mouse models: enhanced genotypes, systemic phenotyping and envirotype modelling. Nat Rev Genet. 2009;10:371-80.

4. Benarroch EE. TRP channels: functions and involvement in neurologic disease. Neurology. 2008;70:648-52.

5. Deng HX, Klein CJ, Yan J, Shi Y, Wu Y, Fecto F, et al. Scapuloperoneal spinal muscular atrophy and CMT2C are allelic disorders caused by alterations in TRPV4. Nat Genet. 2010;42:165-9.

6. Fairbrother WG, Yeh RF, Sharp PA, Burge CB. Predictive identification of exonic splicing enhancers in human genes. Science. 2002;297:1007-13.

7. Fogel BL, Perlman S. An approach to the patient with late-onset cerebellar ataxia. Nat Clin Pract Neurol. 2006;2:629-35. 
8. Hartmann J, Dragicevic E, Adelsberger H, Henning HA, Sumser M, Abramowitz J, et al. TRPC3 channels are required for synaptic transmission and motor coordination. Neuron. 2008;59:392-8.

9. Jen JC, Graves TD, Hess EJ, Hanna MG, Griggs RC, Baloh RW. Primary episodic ataxias: diagnosis, pathogenesis and treatment. Brain. 2007;130:2484-93.

10. Jia Y, Zhou J, Tai Y, Wang Y. TRPC channels promote cerebellar granule neuron survival. Nat Neurosci. 2007;10:559-67.

11. Lin X, Antalffy B, Kang D, Orr HT, Zoghbi HY. Polyglutamine expansion down-regulates specific neuronal genes before pathologic changes in SCA1. Nat Neurosci. 2000;3:157-63.
12. Manto M, Marmolino D. Cerebellar ataxias. Curr Opin Neurol. 2009;22:419-29.

13. Manto MU. The wide spectrum of spinocerebellar ataxias (SCAs). Cerebellum. 2005;4:2-6.

14. Soboloff J, Spassova M, Hewavitharana T, He LP, Luncsford P, $\mathrm{Xu}$ W, et al. TRPC channels: integrators of multiple cellular signals. Handb Exp Pharmacol. 2007;(179):575-91.

15. Tang J, Lin Y, Zhang Z, Tikunova S, Birnbaumer L, Zhu MX. Identification of common binding sites for calmodulin and inositol 1, 4, 5-trisphosphate receptors on the carboxyl termini of trp channels. J Biol Chem. 2001;276:21303-10. 